\title{
SEGURANÇA DAS CONDIÇÕES DE TRABALHO DE TRATORISTA EM APLICAÇÕES DE HERBICIDAS EM SOJA E AMENDOIM E EFICIÊNCIA DE EQUIPAMENTOS DE PROTEÇÃO INDIVIDUAL ${ }^{1}$
}

\section{ADRIANO B. CRISTÓFORO², JOAQUIM G. MACHADO NETO ${ }^{3}$}

RESUMO: Teve-se o objetivo de avaliar a eficiência de um conjunto de equipamentos de proteção individual no controle das exposições proporcionadas ao tratorista aplicando herbicidas nas culturas de soja e de amendoim com o pulverizador de barra e a segurança dessas condições de trabalho. Os pulverizadores utilizados foram os convencionais empregados nas duas culturas para as aplicações de herbicidas em pré-plantio incorporado (ppi), em pré-emergência (pré) e em pósemergência inicial (pós), com volumes de $200 \mathrm{~L} \mathrm{ha}^{-1}$, e $150 \mathrm{~L} \mathrm{ha}^{-1}$ apenas na aplicação em pós, na cultura de soja. As exposições sem EPIs foram de 102,77 mL de calda por dia nas aplicações em ppi, 39,62 em pré e 47,14 em pós-emergência. A eficiência dos EPIs no controle das exposições dérmicas foi de $76,5 \%$ em ppi, $50,9 \%$ em pré e $75,3 \%$ em pós-emergência. Na cultura de soja, foram seguras para o tratorista, sem ou com EPIs, as aplicações de pendimethalin, imazaquin e flumetsulam em ppi; de pendimethalin, acetochlor, clomazone, flumioxazin, imazaquin, metribuzin, sulfentrazone, dimethenamid e flumetsulamem em pré, e de bentazone, glyphosate, imazethapyr, quizalofop-ethyl, chlorimuron ethyl e oxasulfuron em pós. Na cultura de amendoim, sem e com EPIs, foi segura a aplicação de pendimethalin em ppi; em pré, a aplicação de alachlor foi classificada como insegura, sem ou com o uso dos EPIs.

PALAVRAS-CHAVE: herbicidas, soja, amendoim, risco de intoxicação.

\section{SAFETY WORK CONDITIONS FOR TRACTOR OPERATORS IN APPLICATION OF HERBICIDES ON SOYBEAN AND PEANUT CROPS AND EFFECTIVENESS OF PERSONAL PROTECTIVE EQUIPMENT}

\begin{abstract}
The aim of the study was to evaluate the effectiveness of a combination of personal protective equipments (PPE) for the tractor operator applying herbicides on soybean and peanut crops with a tractor mounted boom sprayer and the safetyness of these work conditions. The application of the herbicides were in pre-planting soil-incorporated (ppi), pre-emergence (pre) and post-emergence (post) treatment, with volumes of $200 \mathrm{~L} \mathrm{ha}^{-1}$, except in the post application for soybeans where it was $150 \mathrm{~L} \mathrm{ha}^{-1}$. It was shown that exposure with PPE was $102.77 \mathrm{~mL}$ of spray per day in ppi, 39.62 in pre and 47.14 in post applications. The effectiveness of PPE in controlling dermal exposure was $76.5 \%$ in ppi, $50.9 \%$ in pre and $75.3 \%$ in post applications. With soybeans, were safe for the tractor operator, with or without PPE, the applications of the herbicides pendimethalin, imazaquin and flumetsulam in ppi; pendimethalin, acetochlor, clomazone, flumioxazin, imazaquin, metribuzin, sulfentrazone, dimethenamid and flumetsulam in pre; and bentazone, glyphosate, imazethapyr, quizalofop-ethyl, chlorimuron ethyl and oxasulfuron in post. In peanut crops, were safe the application of pendimethalin ppi, with and without PPE. In pre, the application of alachlor was classified as unsafe with or without the use of PPE.
\end{abstract}

KEYWORDS: herbicides, soybean, peanut, occupational risk.

\footnotetext{
${ }^{1}$ Extraído da Dissertação de mestrado do primeiro autor.

${ }^{2}$ Eng $^{\mathrm{o}}$ Agrônomo, mestre em Agronomia - Entomologia Agrícola, UNESP - Câmpus de Jaboticabal - SP.

${ }^{3}$ Prof. Adjunto, Departamento de Fitossanidade, UNESP - Câmpus de Jaboticabal - SP, joaquim@ fcav.unesp.br. Recebido pelo Conselho Editorial em: 3-3-2005

Aprovado pelo Conselho Editorial em: 21-3-2006
} 


\section{INTRODUÇÃO}

Nas aplicações de herbicidas com o pulverizador de barra acoplado ao trator, nas culturas de soja e de amendoim, como em qualquer outra aplicação de agrotóxico, os tratoristas ficam expostos a riscos de intoxicação. A segurança dessas condições de trabalho deve ser avaliada, e se necessário, medidas de segurança devem ser implementadas. De maneira geral, observa-se que a primeira medida de segurança recomendada para os trabalhadores expostos aos agrotóxicos é o uso de EPIs - equipamentos de proteção individual. Os EPIs são vestimentas individuais que têm eficiência protetora, ou seja, a capacidade de proteger o trabalhador contra o efeito de um fator de processo perigoso e daninho (MILORD \& CASTILLO, 1988). A eficiência protetora dessas vestimentas baseia-se na impermeabilidade ou hidrorrepelência. $\mathrm{O}$ uso dos EPIs causa grande desconforto aos trabalhadores, e as recomendações estão sendo feitas erroneamente com base apenas na classe toxicológica dos agrotóxicos. Dessa forma, a recomendação de uso dos EPIs está incorreta e inadequada, pois os EPIs controlam as exposições dérmicas e respiratórias e não a toxicidade dos agrotóxicos, representada pelas classes toxicológicas.

A eficiência dos EPIs depende dos respectivos princípios de proteção e da parte do corpo que protegem. MOMESSO (2002) avaliou a eficácia de um conjunto de proteção individual feito com tecido hidrorrepelente novo para a proteção do tratorista em aplicações de herbicidas, em préemergência, na cultura de cana-de-açúcar com pulverizador de barra acoplado ao trator. A eficácia dos EPIs foi de $81,6 \%$ de redução da exposição dérmica potencial proporcionada ao tratorista. MACHADO NETO (1997), trabalhando com o pulverizador de barra acoplado em trator, em cultura de algodão, verificou o controle de $50,7 \%$ da exposição potencial proporcionada ao tratorista com um conjunto de EPIs semelhante ao avaliado por MOMESSO (2002). LUNDEHN et al. (1992) e KIECZKA (1993) citam que, em média, as máscaras semifaciais controlam 90\% das exposições dérmicas aos agrotóxicos, e as botas e as luvas de borracha impermeáveis, $95 \%$.

As medidas de segurança no trabalho com agrotóxicos, inclusive o uso de EPIs, devem ser recomendadas com base nos riscos de intoxicação proporcionados pelas condições de trabalho, calculados com os dados de toxicidade do agrotóxico e das exposições proporcionadas pelas condições específicas de trabalho. Essa recomendação de segurança está de acordo com o que preconiza a engenharia de segurança do trabalho e a legislação brasileira sobre esse tema. Na NR-6 (Norma Regulamentadora), aprovada na Portaria $\mathrm{n}^{\mathrm{o}}$ 3.214, de 08-06-1978 (BRASIL, 1978), e na NRR-4 (Norma Regulamentadora Rural), aprovada na Portaria n⿳ํㅜ 3.067, de 12-04-1988 (BRASIL, 1988), em que se determina que os EPIs devem ser recomendados de maneira adequada ao risco. Portanto, antes de se recomendar qualquer medida de segurança no trabalho com agrotóxicos, deve-se avaliar o risco ou a segurança das condições de trabalho com cada agrotóxico utilizado.

Os objetivos deste trabalho foram quantificar as exposições dérmica e respiratória proporcionadas ao tratorista por aplicações de herbicidas nas culturas de soja e amendoim com o pulverizador de barra acoplado em trator; calcular a eficiência de um conjunto de equipamentos de proteção individual no controle destas exposições; calcular a segurança dessas condições de trabalho com os herbicidas recomendados para as duas culturas e classificá-las em seguras ou inseguras; calcular os tempos de trabalho seguro e as necessidades de controle das exposições para as condições classificadas como inseguras.

\section{MATERIAL E MÉTODOS}

As exposições dérmicas e respiratórias proporcionadas pelas condições de trabalho aos tratoristas foram quantificadas em aplicações convencionais de herbicidas nas culturas da soja e do amendoim, em grandes propriedades agrícolas no Estado de São Paulo. Foram avaliadas as exposições potenciais na superfície do corpo dos tratoristas, sem nenhuma medida de proteção, e não controladas pelo conjunto de equipamentos de proteção individual (EPIs) em teste, sob os mesmos, entre os meses de outubro e dezembro de 2001. 
O conjunto de equipamentos de proteção individual foi composto por uma calça comprida com cordel na cintura, camisa de mangas compridas com gola em "V" e fechada com velcro, com cordel na região da cintura, e toca com aba frontal tipo boné e lateral envolvendo a região do pescoço e ombros, e também fechada com velcro embaixo do queixo. Todas as peças foram confeccionadas em tecido de algodão, do tipo brim leve, hidrorrepelente. A hidrorrepelência foi proporcionada pelo tratamento do tecido com Teflon ${ }^{\circledR}$. Esse conjunto foi complementado com viseira de acetato transparente, avental de plástico impermeável, protegendo desde o pescoço até a parte superior das coxas, e luvas impermeáveis de borracha nitrílica. A eficiência das botas de borracha e da máscara descartável foi considerada em 95\% (LUNDEHN et al., 1992 e KIECZKA, 1993).

As aplicações em pré-plantio incorporado ao solo (ppi) foram realizadas com um pulverizador com tanque de $600 \mathrm{~L}$ acoplado ao sistema hidráulico do trator e com uma barra frontal de $3 \mathrm{~m}$ de comprimento. A barra frontal foi afixada no pára-choque do trator e ligada ao circuito hidráulico do pulverizador com uma mangueira de alta pressão. A barra foi composta com seis bicos Teejet 11003 e calibrada para aplicar $200 \mathrm{~L}$ de calda ha ${ }^{-1}$. As aplicações em pré e em pósemergência foram realizadas com um pulverizador com barra convencional, modelo Jacto Condor 600, com barra de $12 \mathrm{~m}$ de comprimento. A barra foi composta com 24 bicos Teejet 11003 e calibrada para aplicar $200 \mathrm{~L}_{\text {de calda }} \mathrm{ha}^{-1}$ em pré e em pós, e $150 \mathrm{~L} \mathrm{ha}^{-1}$ em pós, apenas na cultura de soja. Os tratores estavam com as capotas originais de fábrica.

As exposições proporcionadas em cada condição de trabalho foram avaliadas com dez repetições e em tempos variando entre 20,9 e 32,1 minutos. As exposições respiratórias foram avaliadas durante o triplo dos tempos das dérmicas. As condições atmosféricas durante as avaliações das exposições foram as seguintes: temperatura do ar, variou entre 22,7 e $32,2^{\circ} \mathrm{C}$; umidade relativa do ar, entre 46,8 e $81,1 \%$, e velocidade dos ventos, entre 4,0 e $7,8 \mathrm{~km} \mathrm{~h}^{-1}$.

As exposições dérmicas às caldas aplicadas foram quantificadas com o cátion cobre de um fungicida cúprico (MACHADO NETO \& MATUO, 1989), e as respiratórias, com o cátion Mn de sulfato de manganês (OLIVEIRA \& MACHADO NETO, 2003), ambos adicionados às caldas pulverizadas como traçadores. As exposições dos tratoristas aos herbicidas registrados para as culturas foram calculadas nas quantidades dos mesmos presentes nas quantidades de caldas determinadas nas avaliações das exposições aos traçadores, utilizadas como dados substitutos (JENSEN, 1984). As exposições aos herbicidas foram calculadas com as maiores dosagens recomendadas nas caldas (ANDREI, 1999).

As vestimentas amostradoras das exposições dérmicas utilizadas nas avaliações foram macacões de tecido de algodão do tipo brim grosso, com mangas compridas e com capuz, luvas de algodão e absorventes higiênicos femininos, marca Carefree, de acordo com MACHADO NETO (1990) e MACHADO NETO et al. (1992). O macacão foi utilizado para quantificar as exposições na cabeça + pescoço, tronco (atrás e frente), braços e pernas (atrás e frente), e as luvas, nas mãos. Os absorventes higiênicos femininos foram afixados sobre máscaras semifaciais para quantificar a exposição na face e sobre a parte mediana superior dos pés, as exposições dos pés. As exposições da face e dos pés às caldas foram calculadas por meio da extrapolação das exposições quantificadas nos absorventes para as respectivas áreas superficiais dessas partes do corpo.

As exposições respiratórias foram avaliadas com bombas de fluxo de ar contínuo de uso pessoal, da marca A.P. Buck, reguladas para succionar $2 \mathrm{~L}$ de ar por minuto, de acordo com a metodologia adaptada por OLIVEIRA (2000). Em cada bomba, foi conectada uma mangueira plástica com um cassete na extremidade de $37 \mathrm{~mm}$ de diâmetro interno, contendo um filtro de éster celulose de porosidade de $0,8 \mu$ apoiado sobre uma lâmina circular de celulose.

Após o período de exposição, o macacão, separado em partes (regiões do corpo), as luvas, os absorventes higiênicos femininos e os cassetes foram acondicionados em sacos plásticos identificados e levados para o laboratório. Os filtros e as lâminas de celulose dos cassetes foram transferidos para recipientes de plástico tampados. Nos sacos e recipientes, foi adicionada a solução 
solubilizadora dos cátions traçadores contendo HCL a 0,2 N. Em seguida, amostras foram agitadas manualmente por 30 vezes e permaneceram em repouso por duas horas. Para a quantificação dos cátions, foi filtrada alíquota de $5 \mathrm{~mL}$ em papel de filtro qualitativo de cada solução. As concentrações dos cátions traçadores foram determinadas em um espectrofotômetro de absorção atômica, marca GBC, modelo 932AA.

A curva-padrão do cátion cobre foi preparada a partir da solução de cloreto de cobre $(\mathrm{CuCl})$ Titrisol Merk 9987. A faixa de linearidade foi determinada com as concentrações de 0,$125 ; 0,25$; 0,5; 1,0 e 2,0 ppm $\left(\mathrm{R}^{2}=0,999\right)$. O limite de detecção (LD) determinado foi de $0,017 \mathrm{ppm}$, e o limite de quantificação (LQ), 0,041 ppm. A curva-padrão de manganês foi preparada a partir da solução de cloreto de manganês $(\mathrm{MnCl})$ Titrisol Merk 9988. A faixa de linearidade foi determinada com as concentrações de 0,$125 ; 0,25 ; 0,5 ; 1,0$ e 2,0 ppm $\left(\mathrm{R}^{2}=0,999\right)$. O LD foi de $0,017 \mathrm{ppm}$, e o LQ, 0,041 ppm. Os LDs e LQs foram calculados de acordo com SKOOG et al. (1998).

A segurança dessas condições de trabalho foi avaliada por meio do cálculo da margem de segurança (MS) para cada herbicida registrado nas respectivas épocas de aplicação, com a fórmula:

$$
\mathrm{MS}=(\mathrm{NOEL} 70) / \mathrm{QAE} \times 10
$$

em que,

NOEL - nível de efeito não observado ( $\left.\mathrm{mg} \mathrm{kg}^{-1} \mathrm{dia}^{-1}\right)$, obtido na publicação da TGA (2002);

70 - massa corpórea do trabalhador $(\mathrm{kg}), \mathrm{e}$

QAE - quantidade absorvível da exposição: QAE = 0,1 ED + ER. em que,

ED - exposição dérmica;

ER - exposição respiratória $\left(\mathrm{mg} \mathrm{dia}^{-1}\right)$. As exposições foram extrapoladas para uma jornada de trabalho de $6 \mathrm{~h} \mathrm{dia}^{-1}$, e

10 - fator de segurança para compensar a extrapolação dos dados de toxicidade crônica (NOEL) obtidos em animais de laboratório para o homem (trabalhador).

O critério para classificar a segurança das condições de trabalho foi o seguinte: Se MS $\geq 1$ condição de trabalho segura e risco aceitável e exposição tolerável; se MS < 1 - condição de trabalho insegura, risco inaceitável e exposição intolerável.

Para as condições de trabalho classificadas como inseguras, foi calculada a necessidade de controle das exposições (NCE) com a fórmula proposta por MACHADO NETO (1997):

$\mathrm{NCE}=\left(1-\mathrm{MS}_{<1}\right) 100$.

Para todas as condições de trabalho, também foi calculado o tempo de trabalho seguro (TTS) com as fórmulas propostas por MACHADO NETO (1997:

TTS $=$ MS tee

em que,

tee - tempo de exposição efetiva.

\section{RESULTADOS E DISCUSSÃO}

Os resultados das avaliações das exposições dérmicas nas regiões do corpo e totais, e respiratórias às caldas proporcionadas ao tratorista pelas condições de trabalho de aplicação de herbicidas, em ppi, em pré e pós-emergência das culturas da soja e do amendoim, estão apresentados na Tabela 1. Inicialmente, destaca-se que a quase totalidade das exposições ocorreu na via dérmica e foram insignificantes na via respiratória. Nessas atividades, as exposições respiratórias (ERs) representaram entre 0,010 e 0,040\% das exposições totais. Estes resultados são muito próximos aos 0,003 e $0,021 \%$ da exposição total do tratorista aplicando agrotóxicos em citros com um turbopulverizador arrastado por trator (OLIVEIRA, 2000). 
Verifica-se que as aplicações em ppi proporcionaram exposições potenciais 2,6 vezes maiores que as em pré e 2,2 maiores que as em pós. As exposições em pós-emergência foram apenas 1,2 vez maiores que as em pré. A maior exposição dérmica proporcionada ao tratorista pela aplicação em ppi deveu-se ao fato de que a barra frontal fica na frente e na mesma altura dos pés e pernas do tratorista. Essa barra frontal também fica mais próxima do tratorista que a barra traseira utilizada nas aplicações em pré e pós-emergência. Portanto, com o deslocamento normal do conjunto trator-pulverizador, para fazer a aplicação, as gotas de pulverização que estão dispersas no ar à frente do trator, têm maior possibilidade de atingir os membros inferiores do tratorista.

Nessa atividade e sem o uso dos EPIs, os membros inferiores do tratorista receberam $75,4 \%$ da exposição dérmica total, sendo que $37,7 \%$ dessa exposição atingiu os pés. Com a barra traseira, as exposições dérmicas dos membros inferiores do tratorista foram de $65,4 \%$ do total nas aplicações em pré e 41,6\% em pós-emergência. A menor exposição nos membros inferiores do tratorista, nas aplicações em pós-emergência, pode ser explicada pela retenção das gotas pulverizadas na vegetação formada pelas plantas das culturas e pelas daninhas. $\mathrm{Na}$ aplicação em pré-emergência, devido à ausência da vegetação, as gotas ficam mais disponíveis no ar e podem atingir os membros inferiores do tratorista. Entretanto, as diferenças percentuais não são tão evidentes quanto as diferença quantitativas. As quantidades de caldas determinadas como exposições dérmicas nos membros inferiores do tratorista, nas aplicações em ppi, com a barra frontal, foi de $77,5 \mathrm{~mL} \mathrm{dia}^{-1}$, portanto muito maior que em pré $(25,91)$ e em pós- emergência $(19,62)$.

TABELA 1. Exposições dérmicas nas diversas regiões do corpo e total, e respiratórias $\left(\mathrm{mL} \mathrm{dia}^{-1}\right)$ do tratorista aplicando herbicidas em pré-plantio incorporado (ppi) e em pré e em pós-emergência para o controle de plantas daninhas, em culturas da soja e do amendoim, e eficácia do conjunto de EPIs (\%).

\begin{tabular}{|c|c|c|c|c|c|c|}
\hline \multirow{3}{*}{ Regiões do Corpo } & \multicolumn{4}{|c|}{ Soja e Amendoim - $200 \mathrm{~L} \mathrm{ha}^{-1}$} & \multirow{2}{*}{\multicolumn{2}{|c|}{$\begin{array}{l}\text { Soja - Pós-Emergência } \\
150 \mathrm{~L} \mathrm{ha}^{-1}\end{array}$}} \\
\hline & \multicolumn{2}{|c|}{ Pré-Plantio Incorporado ao Solo } & \multicolumn{2}{|c|}{ Pré-Emergência } & & \\
\hline & Sem EPIs & Com EPIs & Sem EPIs & Com EPIs & Sem EPIs & Com EPIs \\
\hline 1. Cabeça + pescoço & 1,34 & 0,84 & 0,95 & 0,87 & 2,16 & 0,50 \\
\hline 2. Face & 2,05 & 0,21 & 1,39 & 0,15 & 1,84 & 0,19 \\
\hline 3. Mãos & 8,94 & 1,13 & 3,68 & 1,53 & 7,93 & 0,99 \\
\hline 4. Braços & 7,40 & 4,00 & 3,72 & 2,74 & 6,26 & 2,14 \\
\hline 5. Tórax-frente & 3,49 & 1,44 & 2,38 & 1,69 & 3,77 & 1,14 \\
\hline 6. Tórax-atrás & 2,05 & 1,64 & 1,58 & 1,01 & 5,57 & 1,14 \\
\hline 7. Pernas-frente & 23,02 & 6,42 & 9,11 & 5,36 & 6,37 & 3,50 \\
\hline 8. Pernas-atrás & 15,73 & 6,50 & 8,72 & 5,68 & 5,94 & 1,70 \\
\hline 9. Pés & 38,75 & 1,93 & 8,08 & 0,41 & 7,31 & 0,36 \\
\hline Exp. Dérmica & 102,77 & 24,15 & 39,61 & 19,44 & 47,14 & 11,65 \\
\hline Exp. Respiratória & 0,02 & 0,001 & 0,01 & 0,0005 & 0,01 & 0,0005 \\
\hline Total $\left(\mathrm{mL} \mathrm{dia}^{-1}\right)$ & 102,77 & 24,151 & 39,62 & 19,4405 & 47,15 & 11,6605 \\
\hline Eficácia dos EPIs (\%) & \multicolumn{2}{|c|}{76,5} & \multicolumn{2}{|c|}{50,9} & \multicolumn{2}{|c|}{75,3} \\
\hline
\end{tabular}

A eficiência do conjunto de EPIs no controle das exposições dérmicas foi de 76,5\% em ppi, $50,9 \%$ em pré e $75,3 \%$ em pós-emergência. MOMESSO (2002) calculou a eficácia de um conjunto de EPIs similar em $81,6 \%$ da exposição dérmica total proporcionada ao tratorista pulverizando herbicidas na cultura de cana-de-açúcar em pré, com um pulverizador semelhante. O resultado de eficácia do conjunto de EPIs obtido por MOMESSO (2002) é muito superior aos 50,9\% ora obtidos nas aplicações em pré-emergência, porém próximo aos 76,5 obtidos nas aplicações em ppi e aos 75,3\% em pós-emergência. Porém, essas eficácias foram superiores aos 50,7\% de controle da exposição dérmica do tratorista com um conjunto de EPIs, também similarmente avaliada por MACHADO NETO (1997). Esse autor avaliou a exposição proporcionada ao tratorista em aplicações de agrotóxicos com pulverizador de barra convencional em cultura de algodão no estágio de florescimento. 
As distribuições das exposições dérmicas nas regiões do corpo do tratorista (Tabela 1) diferem muito pouco da avaliada por MOMESSO (2002). Esse autor avaliou a exposição dérmica do tratorista aplicando herbicidas em cultura de cana-de-açúcar em pré com o pulverizador de barra montado em trator, com barra de $15 \mathrm{~m}$ de comprimento e bicos tipo leque 11006, modelo Twin Jet. As três regiões do corpo do tratorista mais expostas foram: coxas + pernas frente, mãos e braços. Essas pequenas variações podem ser explicadas pelas diferenças no tamanho das barras e bicos de pulverização e por fatores como a velocidade do vento e a diferença entre os tratores e pulverizadores, dentre outros (HEMMEN, 1992).

Na Tabela 2, verifica-se que, na cultura de soja, foram classificadas como seguras (MS $\geq 1$ ), sem os EPIs, as aplicações em ppi de pendimethalin, imazaquin e flumetsulam; em pré, as aplicações de pendimethalin, acetochlor, clomazone, imazaquin, metribuzin, flumioxazin, sulfentrazone, dimethenamid e flumetsulam; em pós, as aplicações de glyphosate, bentazon, quizalofop-ethyl, clorimuron-ethyl, imazethapyr e oxasulfuron. Sem o uso de EPIs, foram classificadas como inseguras $(\mathrm{MS}<1)$ as aplicações em ppi de trifluralin, metribuzin e cyanazine; em pré, de trifluralin, alachlor, cyanazine e linuron, e em pós, de fenoxapropil-p-ethyl e fomesafen. Com o uso do conjunto de EPIs, tornaram-se seguras as aplicações em ppi de trifluralin e metribuzin; em pré, de trifluralin, e em pós, de fenoxapropil-p-ethyl e fomesafen.

TABELA 2. Exposições potenciais e sob os EPIs, valores de margem de segurança (MS), necessidade de controle da exposição (NCE) e tempo de trabalho seguro (TTS) calculados para os herbicidas aplicados pelos tratoristas, em três épocas de aplicação, nas culturas da soja e de amendoim.

\begin{tabular}{|c|c|c|c|c|c|c|c|c|c|}
\hline \multirow{2}{*}{ Nome Comum } & \multirow{2}{*}{ (g i.a / $100 \mathrm{~L})$} & \multicolumn{2}{|c|}{ Exposição (mg dia) $^{1}$} & \multicolumn{2}{|c|}{ EP } & \multicolumn{2}{|c|}{ Com os EPIs } & \multicolumn{2}{|c|}{ TTS(h) } \\
\hline & & EP & Sob EPIs & MS & $\operatorname{NCE}(\%)$ & MS & $\operatorname{NCE}(\%)$ & EP & EPIs \\
\hline \multicolumn{10}{|c|}{ Soja - aplicações em pré-plantio incorporado ao solo - $200 \mathrm{~L} \mathrm{ha}^{-1}$} \\
\hline Trifluralin & 546,0 & 561,02 & 131,86 & 0,3 & 70 & 1,3 & - & 1,9 & 7,9 \\
\hline Pendimethalin & 750,0 & 770,63 & 181,13 & 1,1 & - & 4,6 & - & 6,5 & 27,8 \\
\hline Imazaquin & 80,5 & 82,71 & 19,44 & 21,1 & - & 88,2 & - & 126,6 & 529,2 \\
\hline Metribuzin & 360,0 & 369,90 & 86,94 & 0,4 & 60 & 1,6 & - & 2,3 & 9,6 \\
\hline Cyanazine & 875,0 & 899,06 & 211,31 & 0,0 & 100 & 0,1 & 90,0 & 0,1 & 0,4 \\
\hline Flumetsulam & 70,0 & 71,93 & 16,91 & 97,1 & - & 404,5 & - & 582,3 & $2.427,0$ \\
\hline \multicolumn{10}{|c|}{ Soja - aplicações em pré-emergência - $200 \mathrm{~L} \mathrm{ha}^{-1}$} \\
\hline Trifluralin & 546,0 & 216,27 & 106,14 & 0,8 & 20 & 1,6 & - & 4,9 & 9,9 \\
\hline Pendimethalin & 750,0 & 297,08 & 145,80 & 2,8 & - & 5,8 & - & 17,0 & 34,5 \\
\hline Acetochlor & $1.680,0$ & 665,45 & 326,59 & 1,1 & - & 2,1 & - & 6,3 & 12,9 \\
\hline Alachlor & $1.680,0$ & 665,45 & 326,59 & 0,1 & 90 & 0,1 & 90,0 & 0,3 & 0,6 \\
\hline Clomazone & 625,0 & 247,56 & 121,50 & 4,0 & - & 8,1 & - & 23,7 & 48,4 \\
\hline Flumioxazin & 60,0 & 23,77 & 11,66 & 8,8 & - & 17,9 & - & 52,8 & 107,1 \\
\hline Imazaquin & 80,5 & 31,89 & 15,65 & 54,7 & - & 111,1 & - & 328,3 & 666,7 \\
\hline Metribuzin & 360,0 & 142,60 & 69,98 & 1,0 & - & 2,0 & - & 5,9 & 12,0 \\
\hline Linuron & 742,5 & 294,10 & 144,34 & 0,3 & 70 & 0,6 & 40,0 & 1,8 & 3,6 \\
\hline Sulfentrazone & 300,0 & 118,83 & 58,32 & 5,9 & - & 12,0 & - & 35,3 & 71,9 \\
\hline Cyanazine & 875,0 & 346,59 & 170,10 & 0,0 & 100 & 0,1 & 90,0 & 0,2 & 0,5 \\
\hline Dimethenamid & 562,5 & 222,81 & 109,35 & 1,9 & - & 3,8 & - & 11,3 & 23,0 \\
\hline Flumetsulam & 70,0 & 27,73 & 13,61 & 251,6 & - & 510,7 & - & $1.509,3$ & $3.063,9$ \\
\hline \multicolumn{10}{|c|}{ Soja - aplicações em pós-emergência - $150 \mathrm{~L} \mathrm{ha}^{-1}$} \\
\hline Bentazone & 480,0 & 226,27 & 55,92 & 3,1 & - & 12,5 & - & 18,6 & 75,0 \\
\hline Fomesafen & 100,0 & 47,14 & 11,65 & 0,4 & 60 & 1,5 & - & 2,2 & 8,9 \\
\hline Glyphosate & $1.920,0$ & 905,09 & 223,68 & 2,3 & - & 9,4 & - & 13,9 & 56,3 \\
\hline fenoxaprop-ethyl & 73,3 & 34,55 & 8,54 & 0,8 & 20 & 3,2 & - & 4,8 & 19,4 \\
\hline Imazethapyr & 70,6 & 33,28 & 8,22 & 578,8 & - & $2.320,7$ & - & $3.472,7$ & $13.924,5$ \\
\hline quizalofop-ethyl & 33,3 & 15,70 & 3,88 & 5,5 & - & 22,0 & - & 33,2 & 131,9 \\
\hline chlorimuron ethyl & 13,3 & 6,27 & 1,55 & 137,4 & - & 530,5 & - & 824,2 & $3.182,9$ \\
\hline Oxasulfuron & 500,0 & 235,70 & 58,25 & 2,5 & - & 10,0 & - & 14,8 & 59,7 \\
\hline \multicolumn{10}{|c|}{ Amendoim - aplicações em pré-plantio incorporado ao solo - $200 \mathrm{~L} \mathrm{ha}^{-1}$} \\
\hline Trifluralin & 546 & 561,02 & 131,86 & 0,31 & 69 & 1,32 & - & 1,87 & 7,94 \\
\hline Pendimethalin & 750 & 770,63 & 181,13 & 1,09 & - & 4,63 & - & 6,54 & 27,76 \\
\hline \multicolumn{10}{|c|}{ Amendoim - aplicações em pré-emergência - $200 \mathrm{~L} \mathrm{ha}^{-1}$} \\
\hline Alachlor & 1680 & 665,45 & 326,59 & 0,05 & 95 & 0,11 & 89,0 & 0,32 & 0,64 \\
\hline
\end{tabular}


Na cultura de amendoim, sem o uso de EPIs, foram classificadas como inseguras $(\mathrm{MS}<1)$ as aplicações em ppi de trifluralin, e em pré, de alachlor. Com o uso do conjunto de EPIs, apenas a aplicação do trifluralin tornou-se segura.

De acordo com esses resultados, verifica-se que a primeira medida de segurança a ser adotada é a seleção e a recomendação apenas dos herbicidas cujas aplicações sem a proteção do conjunto de EPIs foram classificadas como seguras (MS > 1). Nessas condições, os EPIs devem ser usados como medidas de segurança preventiva quanto à possibilidade de acidente de trabalho, que pode resultar em altas exposições do trabalhador. As recomendações do uso dos EPIs e do número de EPIs devem ser feitas com base na necessidade de controle da exposição calculada para a condição de trabalho. Os primeiros EPIs a serem usados devem ser aqueles que protegem as regiões do corpo do trabalhador que recebem as maiores exposições. Para o tratorista, nas atividades estudas, são aqueles EPIs que protegem os pés, pernas e coxas, que receberam as maiores exposições dérmicas (Tabela 1).

Como medida de proteção, o conjunto de EPIs avaliado foi eficiente nas aplicações de poucos herbicidas (Tabela 1). As exposições dos pés dos tratoristas foram as mais controladas, principalmente porque a eficiência das botas foi considerada como 95\% (LUNDEHN et al., 1992, e KIECZKA, 1993).

Como medida de segurança coletiva, a restrição do tempo de exposição ao TTS pode ser utilizada apenas quando o valor calculado for maior ou igual à metade do tempo de uma jornada diária de trabalho. A restrição da exposição ao TTS calculado ainda pode ser combinada com o uso de EPIs.

\section{CONCLUSÕES}

As exposições sem EPIs foram de 102,77 mL de calda por dia nas aplicações em ppi, 39,62 em pré e 47,14 em pós-emergência.

A eficiência dos EPIs no controle das exposições dérmicas foi de 76,5\% em ppi, 50,9\% em pré e $75,3 \%$ em pós-emergência.

$\mathrm{Na}$ cultura de soja, foram seguras para o tratorista as aplicações em ppi, sem ou com EPIs, dos herbicidas de pendimethalin, imazaquin e flumetsulam, e inseguras sem EPIs as de trifluralin, metribuzin e cyanazine. O uso dos EPIs tornou segura as aplicações de trifluralin e metribuzin. Em pré-emergência, foram seguras, sem ou com EPIs, as aplicações de pendimethalin, acetochlor, clomazone, flumioxazin, imazaquin, metribuzin, sulfentrazone, dimethenamid e flumetsulam, e inseguras as de trifluralin, alachlor, linuron e cyanazine. $\mathrm{O}$ uso dos EPIs tornou segura apenas a aplicação de trifluralin; em pós, foram seguras as aplicações de bentazone, glyphosate, imazethapyr, quizalofop-ethyl, chlorimuron ethyl e oxasulfuron, e inseguras as de fomesafen e fenoxaprop-ethyl, que se tornaram seguras com o uso dos EPIs.

Na cultura de amendoim, em ppi, sem e com EPIs, foi segura a aplicação de pendimethalin, e insegura a aplicação de trifluralin sem EPIs , mas se tornou segura com o uso dos EPIs. Em pré, a aplicação de alachlor foi classificada como insegura, sem ou com o uso dos EPIs.

\section{AGRADECIMENTOS}

À FAPESP, pela concessão de bolsa de mestrado ao primeiro autor e de auxílio à pesquisa.

\section{REFERENCIAS}

ANDREI, E. Compêndio de defensivos agrícolas: guia prático de produtos fitossanitários para uso agrícola. 6.ed. São Paulo: Organização Andrei Editora, 1999. 672 p.

BRASIL. Ministério do Trabalho. Norma Regulamentadora $\mathrm{n}^{\mathrm{0}}$ 6, Portaria $\mathrm{n}^{\mathbf{0}} 3.214$, de 08 de jun. 1978. Diário Oficial da União, Brasília, 1978. 
BRASIL. Ministério do Trabalho. Norma Regulamentadora Rural $\mathrm{n}^{\mathrm{o}}$ 4, Portaria $\mathrm{n}^{\mathrm{o}}$ 3.067, de 12 de abr. 1988. Diário Oficial da União, Brasília, abr. 1988.

HEMMEN, J.J. Van. Agricultural pesticide exposure data bases for risk assessment. Reviews of Environmental Contamination and Toxicology, New York, v.126, p.85, 1992.

JENSEN, J.K. The assumptions used for exposure assessments. In: SIEWIERSKI, M. (Ed.). Determination and assessment of pesticide exposure. New York: Elsevier, 1984. p.147-52.

KIECZKA, H. Requirements for safe-guarding the health of applicators of plant protection products - an overview. In: INTERNATIONAL SYMPOSIUM ON PESTICIDES APPLICATION TECHNIQUES, 2., 1993, Strasbourg. Annales... v.2/2, p.455-462.

LUNDEHN. J.; WESTPHAL, D.; KIECZKA, H.; KREBS, B.; LÖCHER-BOLZ, S.; MAASFELD, W.; PICK, E.D. Uniform principles for safeguarding the health of applicators of plant protection products (Uniform principles for operator protection). Berlin: Kommissionsverlag Paul Parey, 1992. $90 \mathrm{p}$.

MACHADO NETO, J.G. Quantificação e controle da exposição dérmica de aplicadores de agrotóxicos na cultura estaqueada de tomate, na região de Cravinhos - SP. 1990. $112 \mathrm{f}$. Tese (Doutorado em Agronomia) - Faculdade de Ciências Agrárias e Veterinárias, Universidade Estadual Paulista, Jaboticabal, 1990.

MACHADO NETO, J.G. Estimativa do tempo de trabalho e da necessidade de controle da exposição dos aplicadores de agrotóxicos. 1997. 83 f. Tese (Livre-Docência em Agronomia) Faculdade de Ciências Agrárias e Veterinárias, Universidade Estadual Paulista, Jaboticabal, 1997.

MACHADO NETO, J.G.; MATUO, T. Avaliação de um amostrador para o estudo da exposição dérmica potencial de aplicadores de defensivos agrícolas. Ciência Agronômica, Jaboticabal, v.4, n.2, p.21-2, 1989.

MACHADO NETO, J.G.; MATUO, T.; MATUO, Y.K. Dermal exposure of pesticide applicators in staked tomato (Lycopersicon esculentum) crops: efficiency of a safety measure in the application equipment. Bulletin of Environmental Contamination and Toxicology, New York, v.48, p.529-34, 1992.

MOMESSO, J.C. Influência dos períodos e volumes de aplicação na segurança dos tratoristas em aplicações de herbicidas na cultura de cana-de-açúcar (Saccharum spp.). 2002. 56 f. Dissertação (Mestrado em Produção Vegetal) - Faculdade de Ciências Agrárias e Veterinárias, Universidade Estadual Paulista, Jaboticabal, 2002.

MILORD, D.R.; CASTILLO, P. Glosario de términos en salud ambiental con especial énfasis en los efectos en salud relacionados con las sustancias quimicas. México: Centro Panamericano de Ecología Humana y Salud, 1988. 75 p.

OLIVEIRA, M.L. Segurança no trabalho de aplicação de agrotóxicos com turboatomizador e pulverizador de pistolas em citros, na região de Jaboticabal - SP. 2000. 99 f. Dissertação (Mestrado em Produção Vegetal) - Faculdade de Ciências Agrárias e Veterinárias, Universidade Estadual Paulista, Jaboticabal, 2000.

OLIVEIRA, M.L.; MACHADO NETO, J.G. Use of manganese as tracer in the determination of respiratory exposure and relative importance of exposure routes in the safety of pesticide applicators in citrus orchards. Bulletin of Environmental Contamination and Toxicology, New York, v.70, p.41521, 2003.

SKOOG, D.A.; HOLLER, F.J.; NIEMAN, T.A. Principles of instrumental analysis. $5^{\text {th }}$ ed. Philadelphia: Saunders College, 1998. 435 p.

TGA - Therapeutic Goods Administration Commonwealth Department of Health and Ageing. ADI list: acceptable daily intakes for agricultural and veterinary chemicals. Canberra: TGA, 2002. 39 p. 